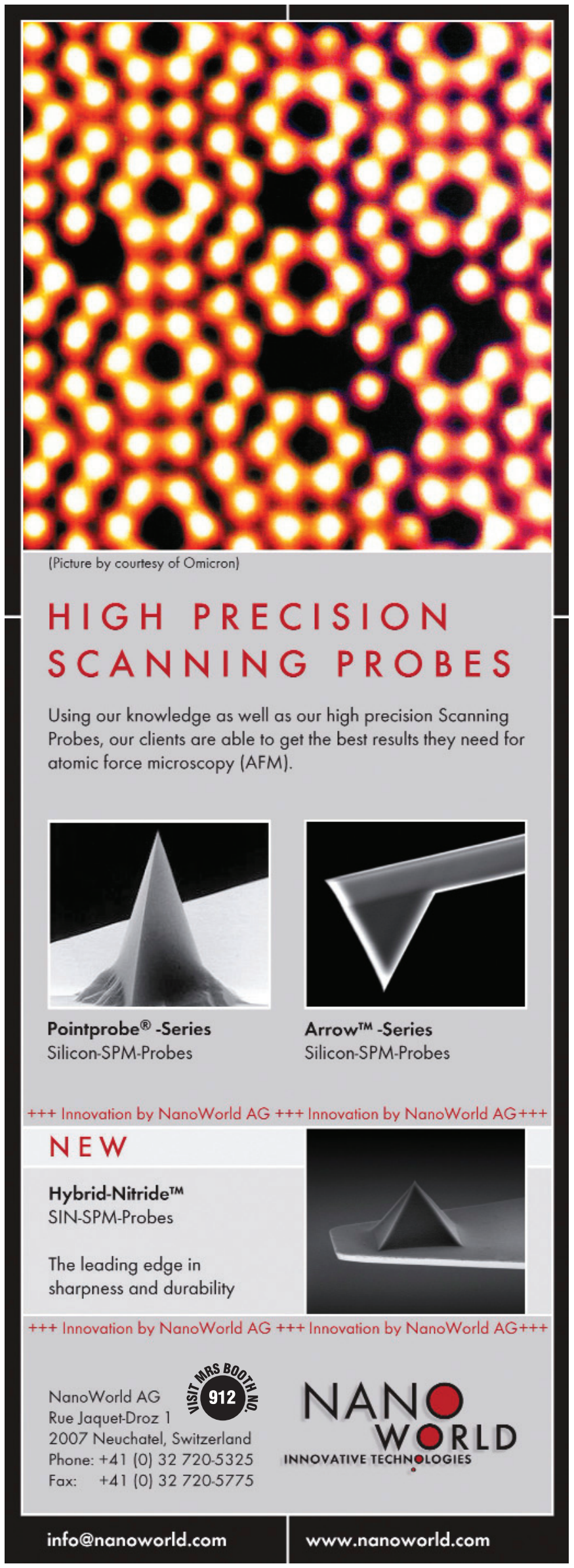

and L. Huang from the Shanghai Institute of Ceramics of the Chinese Academy of Sciences. Their technique ensures a uniform structure on the ceramic reticule.

As reported in the July issue of the Journal of the American Ceramic Society (p. 1392), the researchers prepared a slurry from silicon nitride powders mixed with $7 \mathrm{wt} \%$ alumina added as a sintering additive, $23 \mathrm{wt} \%$ silica sol as a binder, $1.5 \mathrm{wt} \%$ carboxymethylcellulose as a thickening agent, and $0.2 \mathrm{wt} \%$ of Nopco 267-A as an antifoaming agent. The slurry was ball milled using silicon nitride balls. A polyurethane sponge was submerged in slurry and compressed there to achieve the most adsorption possible, then centrifuged at high speed. In this manner, the lattice was uniformly covered with a thin film. The preform thus obtained was dried and recoated in slurry, this time using vacuum to ensure coverage. This process was repeated using slurries of different viscosities.

The measured weight gain of the sample after the first coating in slurry slightly increased with increasing slurry viscosity, and became constant for high-viscosity slurries. However, for the second coating, the measured weight gain was double that of the first coating for low-viscosity slurries, and increased dramatically with slurry viscosity. This is an effect of the enhanced adsorption created by the first thin film deposited on the lattice, said the researchers.

Measurements of the diameters in the lattice struts were fairly uniform and almost identical to the joint diameters. The strut diameter increased with increasing slurry viscosity, as expected, since the weight gain was higher at higher viscosity in the second coating. Through this process, the researchers demonstrated the ability to obtain a highly uniform and robust reticule structure, even starting with any irregularly shaped sample. The combination of highspeed centrifuging after each coating and the use of high-viscosity slurry for the second coating improved mechanical properties of RPCs, the researchers reported. The only difficulty they noted was the need for completing the second coating before drying the sample to prevent it from absorbing water and crashing.

SIARI SOSA

\section{Circuit Quantum Electrodynamics Used to Couple Photon with Qubit}

One of the goals in quantum physics is to develop a quantum computer in which the transistors that form logic gates to manipulate bits in a classical computer are replaced with quantum logic gates that can store and process information quantum mechanically. The fundamental units of information manipulated in a quantum computer are called quantum bits or "qubits," but implementations of the quantum logic gates are sometimes also referred to as qubits. R. Schoelkopf and the theory group of S. Girvin, both at Yale University, have conducted an experiment in which a single photon was coherently coupled to a single superconducting qubit or artificial atom consisting of about 10 billion aluminum atoms acting in concert like a single atom (see Figure 1). One way to connect qubits in a quantum computer is to make a "quantum bus" to carry information back and forth by using single photons. The work of Schoelkopf, Girvin, postdoctoral associate A. Wallraff, and graduate student D. Schuster introduces a paradigm in which quantum optics experiments can be performed in a microchip electrical circuit using microwaves instead of visible photons and lasers.

As reported in the September 9 issue of Nature (p. 162), the researchers constructed a miniaturized superconducting cavity based upon a coplanar waveguide structure of niobium on a silicon substrate in which to trap the microwave photon and force it to collide about 12 million times per second with the qubit implementation based upon a Cooper pair box. The Cooper pair box consisted of a long, thin superconducting island (several microns

For more information, see http://advertisers.mrs.org 


\section{Know the}

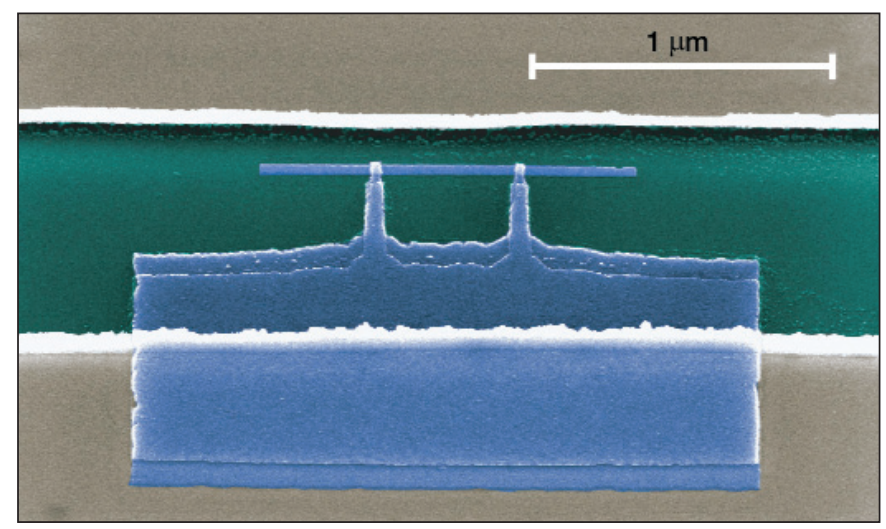

\section{Ups and Downs ...}

\section{Steep slope}

+ Big height difference

+ Large scan field

\section{The LSM S PASCAL confocal laser scanning microscope} broadens your microscopic horizons.

Figure 1. Integrated circuit for cavity quantum electrodynamics: false color electron micrograph of a Cooper pair box (blue) fabricated onto the Si substrate (green) into the gap between the center conductor (top) and the ground plane (bottom) of a resonator (beige) using electron beam lithography and double angle evaporation of aluminum. The Josephson tunnel junctions are formed at the overlap between the long thin island parallel to the center conductor and the fingers extending from the much larger reservoir coupled to the ground plane. Reprinted with permission from Nature $\mathbf{4 3 1}$ (September 9, 2004) p. 163. (02004 Nature Publishing Group.

long and $<1 \mu \mathrm{m}$ wide) fabricated in the gap between the center conductor of the coplanar waveguide structure and the ground plane. The Cooper pair box was coupled by two submicron Josephson junctions to a larger superconducting reservoir, which was coupled to the ground plane. Under the rules of quantum mechanics, the state of the system becomes a coherent superposition of two simultaneous possibilities: The energy is either an excitation of the atom or it is a photon. It is this superposition that was observed in the Yale experiment.

The researchers said, "The very strong coupling of the microwaves to the qubit... makes it possible to use probe photons sent through the cavity to quickly and accurately determine whether the artificial atom is in its ground state or excited state without changing that state." This type of measurement is important in developing a quantum computer. The researchers said, "Such a device [quantum computer] consists of quantum bits that, unlike ordinary bits, can be in coherent superpositions of 0 (ground state) or 1 (excited state) at the same time."

In order to isolate one photon, the researchers operated the circuit at a temperature of 0.02 kelvin. They said, "This also allowed us to build the circuit with superconducting wires that have no resistance, so we could make a cavity where the single photon could be stored for a relatively long time."

\section{Miniature Fuel Cell Fabricated Using Microelectronic Techniques Displayed a Porous Si Layer as a Catalyst Support Layer}

Several types of miniature fuel cells are in development, with a primary goal of utilizing current microfabrication technology to achieve power generation for portable electronic devices. Many of these miniature fuel cells use catalyst layers containing $\mathrm{Pt} / \mathrm{Ru}$ on activated carbon, which is incompatible with the silicon-batch fabrication process. In order to better implement Si microfabrication techniques to assemble miniature fuel cells, M. Hayase, T. Kawase, and T. Hatsuzawa from the Tokyo Institute of Technology in Japan developed an alternative process to forming a catalyst layer, which these researchers described in the August issue of Electrochemical and Solid-State Letters (p. A231).

Highly resolved images. Simple and efficient. Nan-destruxtive.

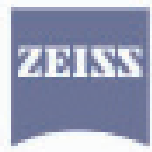

We make it visible 